\title{
Partial Dislocations in Graphene and their Atomic Level
}

\section{Migration Dynamics}

Alex W. Robertson, ${ }^{1 *}$ Gun-Do Lee, ${ }^{2}$ Kuang He, ${ }^{1}$ Ye Fan, ${ }^{1}$ Christopher S. Allen, ${ }^{1}$ Sungwoo Lee, ${ }^{2}$ Heeyeon Kim, ${ }^{3}$ Euijoon Yoon, ${ }^{2}$ Haimei Zheng, ${ }^{4}$ Angus I. Kirkland, ${ }^{1}$ and Jamie H. Warner ${ }^{1 *}$

${ }^{1}$ Department of Materials, University of Oxford, Parks Road, Oxford, OX1 3PH, United Kingdom

${ }^{2}$ Department of Materials Science and Engineering, Seoul National University, Seoul, Korea

${ }^{3}$ Energy Materials Laboratory, Korean Institute of Energy Research, 152 Gajeong-ro, Yuseong-gu, Daejeon 305-343, Korea

${ }^{4}$ Materials Science Division, Lawrence Berkeley National Lab, Berkeley, California 94720, United States

*alex.robertson2@materials.ox.ac.uk; *jamie.warner@materials.ox.ac.uk;

\section{abstract}

We demonstrate the formation of partial dislocations in graphene at elevated temperatures of $\geq 500{ }^{\circ} \mathrm{C}$ with single atom resolution aberration corrected transmission electron microscopy. The partial dislocations spatially redistribute strain in the lattice, providing an energetically more favorable configuration to the perfect dislocation. Low-energy migration paths mediated by partial dislocation formation have been observed, providing insights into the atomistic dynamics of graphene during annealing. These results are important for understanding the high temperature plasticity of graphene and partial dislocation behavior in related crystal systems, such as diamond cubic materials.

KEYWORDS: graphene, TEM, dislocations, defects 
Dislocations are underpinning defects that govern many mechanical properties in materials, and understanding their behavior is crucial for fundamental materials processing techniques such as work hardening and annealing. ${ }^{1,2}$ The underpinning role of dislocations in determining material properties is particularly acute in relation to their governance of plastic deformation, which is mediated by the formation, motion and annihilation of dislocations. For many materials dislocations will dissociate into a pair of Shockley partial dislocations, extending the zero-dimensional, point defect core of a perfect dislocation into an extended ribbon defect, a stacking fault along which kinks or jogs may reside, that is bounded by the partial dislocation pair. The dissociation reduces the local strain in the crystal lattice and enables dislocation migration through alternative mechanisms, such as kink assisted diffusion. ${ }^{1}$ These migration mechanisms are of particular interest in annealing processes, where elevated temperatures lead to greater dislocation mobility and annihilation, thus reducing the dislocation density. Manipulation of the dislocation density is the single most important factor in controlling the mechanical properties of metals and other materials, and as such is a crucial aspect of material processing. ${ }^{2}$

Transmission electron microscopy (TEM) has been employed extensively to study the behavior of dislocations. However, the inherent difficulties of interpreting a 3D structure from a projected 2D image has historically precluded the unambiguous determination of single atom position and bonding; images are instead of single atomic columns, impeding identification of atomic level dynamics. ${ }^{3-6}$ Monolayer graphene, with its single atom thickness, is an ideal material system for direct TEM imaging of atomic structure. ${ }^{7}$ The two-dimensional nature of graphene precludes the existence of screw dislocations, leaving only edge dislocations as the origin of plastic deformation. ${ }^{8-11}$ These edge dislocations, together with their glide and climb dynamics, were recently imaged at single atom sensitivity. ${ }^{12}$ Their formation and annihilation has been characterized, ${ }^{13,14}$ and the rippling associated with the addition of dislocation cores has been shown to assist in strain relief in the graphene lattice through out-of-plane distortions. ${ }^{15}$ However, the observation or prediction of partial dislocations in monolayer graphene has not been reported, with experiments limited to the observation of partials in bilayer graphene, where the system 
behaves as the thinnest exemplar of three-dimensional graphite. ${ }^{16}$ Moreover direct single atom imaging of the migration dynamics of partials are lacking for any material system. ${ }^{5,17}$ This lack of experimental data is of particular relevance not just for graphene, but also for understanding dislocation partials in materials extensively used in the semiconductor industry, including strained silicon and germanium, in which they affect electronic performance by acting as sites of charge scattering and trapping. ${ }^{18}$ Such data is also of interest from a fundamental metallurgical perspective, where it may lead to a better understanding of the atomistic mechanisms relevant to annealing processes.

In this work we use low-voltage aberration corrected transmission electron microscopy (AC-TEM) with an in-situ temperature controlled sample holder to reveal the atomic structure and dynamics of partial dislocations in graphene. Their formation was found to be temperature dependent, with partial dislocations forming at $500{ }^{\circ} \mathrm{C}$ and above. Climb and glide mechanisms of the partials have been captured, demonstrating unique behavior to that found for perfect dislocations.
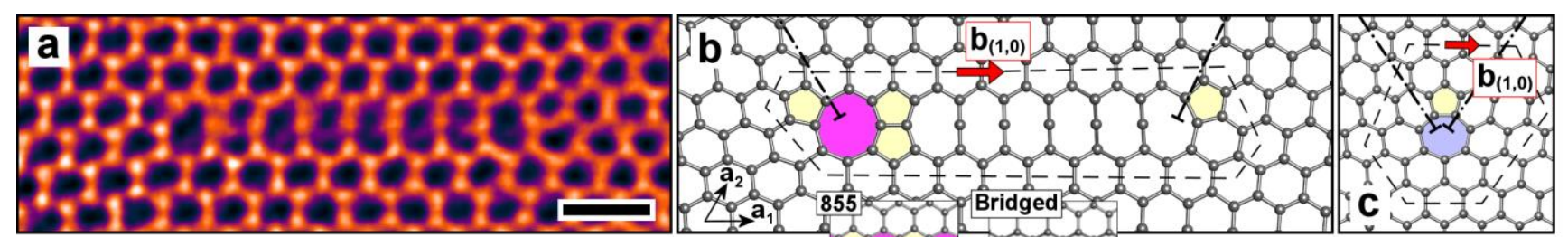

d

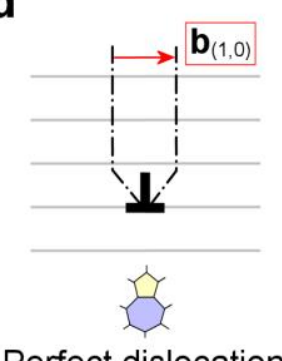

Perfect dislocation e

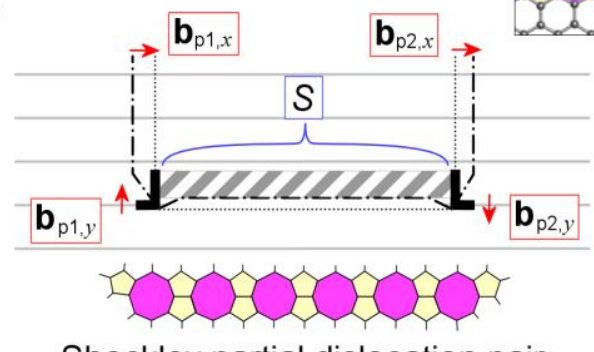

Shockley partial dislocation pair
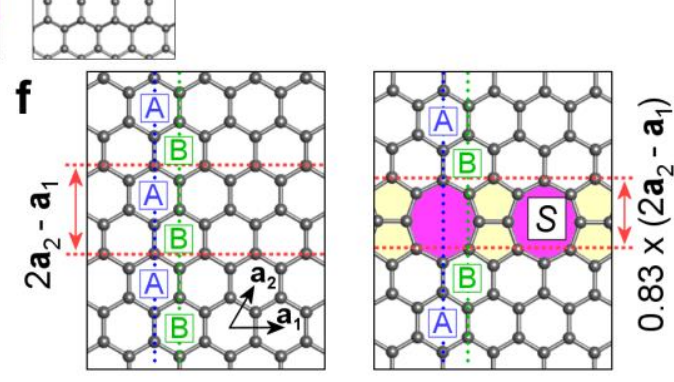

Figure 1. (a) AC-TEM of a partial dislocation pair in graphene formed at $700{ }^{\circ} \mathrm{C}$. Bright spots directly correspond to atomic positions. Image processing details are given in Figure S2. Scale bar is $0.5 \mathrm{~nm}$. (b)

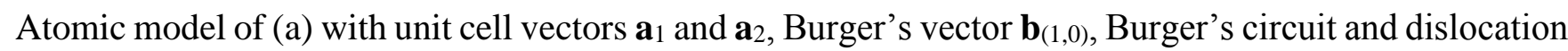
axes marked. The inserts discriminate between reconstructed (855) and non-reconstructed (bridged) bonding. (c) Atomic model of the equivalent $\mathbf{b}_{(1,0)}$ perfect dislocation. (d) Graphic illustrating the perfect dislocation with Burger's vector $\mathbf{b}_{(1,0)}$. (e) Graphic of a $\mathbf{b}_{(1,0)}$ partial dislocation pair described by Burger's 
vectors $\mathbf{b}_{\mathrm{p} 1}$ and $\mathbf{b}_{\mathrm{p} 2}$, separated by a stacking fault, $S$. (f) Atomic models illustrating the in-plane stacking fault generated by the 855 line defect. The perfect lattice exhibits AB stacking, however the inclusion of a bisecting 855 line defect leads to AA stacking. The experimentally measured lattice contraction along direction $2 \mathbf{a}_{2}-\mathbf{a}_{1}$ is also shown.

Extended line defects were frequently observed in the graphene lattice when heated to temperatures of $500{ }^{\circ} \mathrm{C}$ and above (Figure 1a), with their formation typically occurring at defect seeds before detaching, leaving an isolated partial dislocation pair (Figure S1, Movie S1). The body of these line defects act as in-plane stacking faults, whilst the terminations at each end of the line are Shockley partial dislocation cores (Figures S3 and S4). The partial dislocation pair in Figure 1a has a Burger's vector of $\mathbf{b}_{(1,0)}$ (Figure 1b), with the index $(1,0)$ indicating the direction relative to the graphene unit cell base vectors $\left(\mathbf{a}_{1}, \mathbf{a}_{2}\right){ }^{9}$ The connected partial pair are the equivalent to a single perfect dislocation, with the equivalent $\mathbf{b}_{(1,0)}$ perfect dislocation shown for comparison in Figure 1c. An alternative partial dislocation structure with similar characteristics to that in Figure 1a was also observed (Figures S5-S8).

Figures 1d and e summarize the key structural differences between a perfect dislocation and a partial dislocation pair. The perfect dislocation is a point defect, whereas the equivalent pair of Shockley partials are separated by an extended one-dimensional line defect; a twinning boundary that can alternatively be interpreted as in-plane stacking fault that alters the lattice from AB to AA stacking (Figure 1f and Figure S9). This line defect has an 855-ring bonding arrangement, ${ }^{19,20}$ but was also observed in the nonreconstructed bridging configuration (Figure $1 \mathrm{~b}$ inserts), described in more detail elsewhere. ${ }^{21}$ Discriminating between the two configurations was possible from bond length measurements (Figure S10), which show a $(49 \pm 5)$ pm difference in the inter-carbon distance for the two structures. The stacking fault causes a lattice contraction of $\sim 17 \%$ across the defect, as measured directly from AC-TEM images (Figure S11). This measurement confirms that the Frank's energy criterion ${ }^{1}$ for dislocation dissociation is satisfied for the observed partials, the calculations for which are shown in the Supporting Information. 

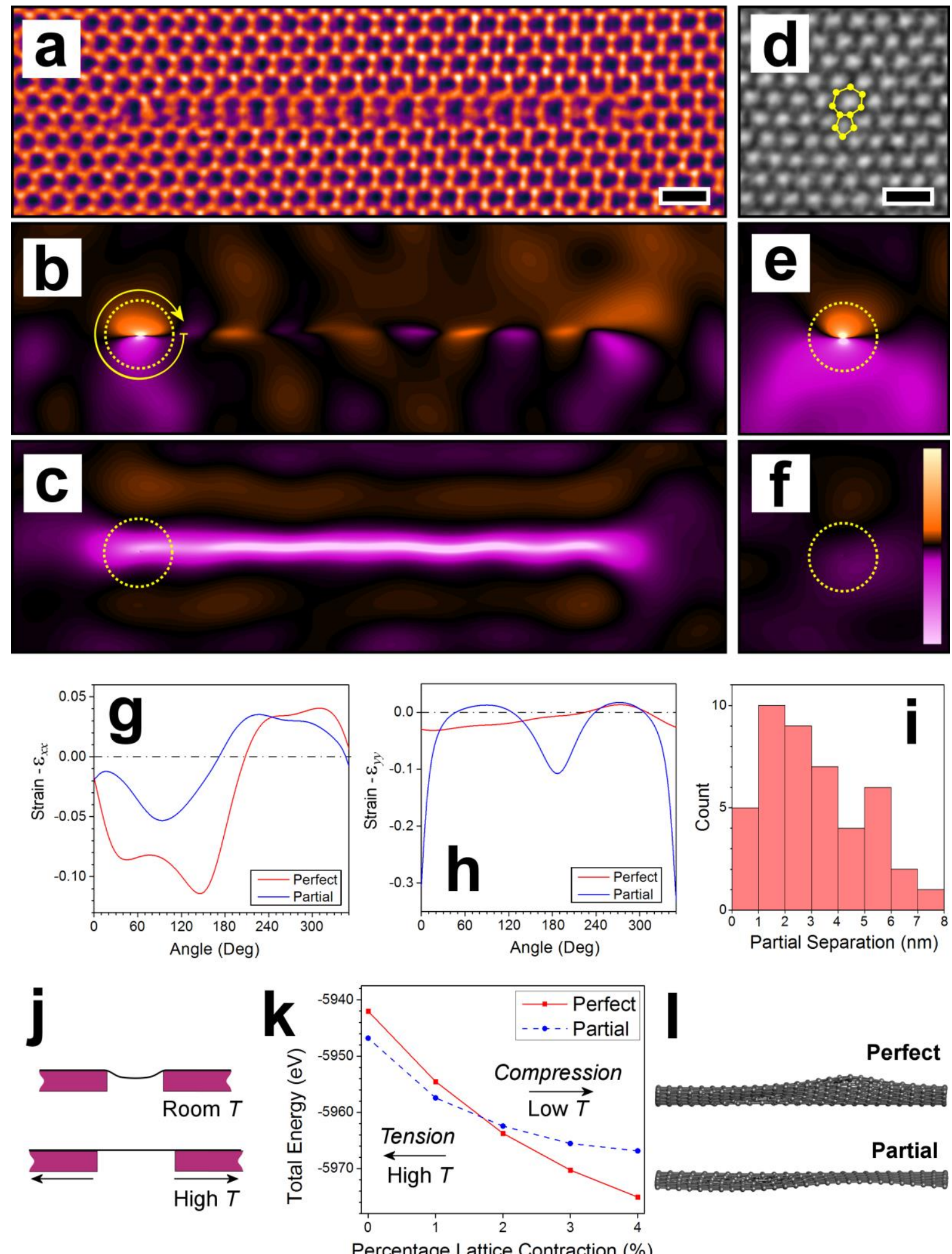

Perfect
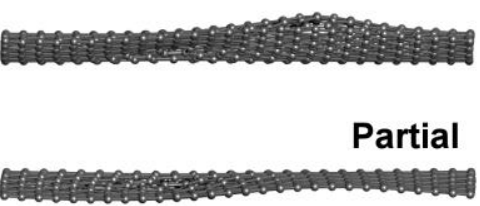

Percentage Lattice Contraction (\%)

Figure 2. (a) AC-TEM image $\left(700^{\circ} \mathrm{C}\right)$ and $(b, c)$ GPA calculated $\varepsilon_{x x}$ and $\varepsilon_{y y}$ strain maps of a partial dislocation pair. (d) AC-TEM image (room temp.) and (e,f) GPA calculated $\varepsilon_{\mathrm{xx}}$ and $\varepsilon_{\mathrm{yy}}$ strain maps of a perfect dislocation. Scale bars are $0.5 \mathrm{~nm}$. Strain color scale is +0.5 (white/yellow) to -0.5 (white/blue). 
Radial intensity profiles of (g) the $\varepsilon_{\mathrm{xx}}$ strain component and (h) the $\varepsilon_{\mathrm{yy}}$ strain component for the perfect and partial dislocation. (i) Distribution of partial dislocation separations (stacking fault lengths). (j) Schematic of suspended graphene at room temperature (top) and high temperature (bottom), with thermal expansion of the supporting $\mathrm{Si}_{3} \mathrm{~N}_{4}$ putting the graphene in tension. (k) DFT calculated energies for perfect and partial dislocation structures for different levels of lattice contraction. (1) Profile view of DFT geometry optimized perfect and partial dislocation structures.

The favorability for partial dislocation formation, as established by Burger's vector analysis and adherence to Frank's criterion, is further supported by strain analysis of the defect. Geometric phase analysis (GPA) ${ }^{22}$ from images of partial and perfect dislocations (Figures 2a-f) provides lattice phase maps and components of the symmetric strain tensor (Figures S13-S16). In general, partials form to reduce the strain energy, redistributing the strain from a single, high strain point defect along a line. This can be seen from the $\varepsilon_{x x}$ strain components, which show a lower lattice strain for the partial dislocation core (Figure 2g). Selecting the $\varepsilon_{x x}$ component ensures that there is a negligible strain contribution to the component from the stacking fault as analysis of the other strain components, such as $\varepsilon_{y y}$ (Figure 2c), demonstrates that the stacking fault itself generates significant strain (Figure $2 \mathrm{~h}$ ). The energetic favorability for partial dislocation formation is broadly a competition between the energy gain arising from dissociation of a perfect dislocation into partials, such as from strain reduction and bonding reconstructions, and the energy cost of generating the linking stacking fault, the stacking fault energy (SFE). ${ }^{1}$ This latter energy cost acts to limit the stacking fault length, and hence the partial dislocation separation. Our data show a steady reduction in the observed separation length to a maximum of $\sim 8 \mathrm{~nm}$ (Figure 2i), although greater separations can be achieved through the formation of $60^{\circ}$ lattice switches in the stacking fault line (Figure S17). The relative magnitude of the SFE also provides an explanation for the temperature dependence of partial dislocation formation; if the SFE were to have an inverse temperature dependence ${ }^{23}$ partial dislocation formation would only be favored at higher temperatures, when the SFE cost is sufficiently low to be offset by the energy saving due to dissociation. 
To explain this inverse temperature dependence it is necessary to consider two factors: (i) the planar nature of graphene allows the formation of out-of-plane distortions to release strain, as has been observed for perfect dislocations in graphene; ${ }^{15}$ (ii) the thermal expansion coefficient of graphene is several orders of magnitude smaller than that of the supporting $\mathrm{Si}_{3} \mathrm{~N}_{4}$ thin-film $\left(10^{-6} \mathrm{~K}^{-1}\right.$ for silicon nitride thin films ${ }^{24}$ vs. $10^{-8} \mathrm{~K}^{-1}$ at $700{ }^{\circ} \mathrm{C}$ for graphene ${ }^{25}$ ), thus graphene suspended across the membrane holes experiences increasing biaxial tension with rising temperature (Figure $2 \mathrm{j}$ ) due to the biaxial expansion of the $\mathrm{Si}_{3} \mathrm{~N}_{4}$ support. This has been clearly demonstrated with SEM and AFM measurements by Bao et al. ${ }^{26}$ Overall, the combination of these two factors suggests that rises in temperature would increase the formation energy of perfect dislocations, due to the stiffening of the graphene sheet inhibiting the out-of-plane distortions required to reduce the strain energy. DFT calculations performed for various levels of lattice contraction, to imitate the stiffening effect at increased temperature, show that the partial dislocation stacking fault structure incurs less of an energy penalty under stiffer conditions (Figure 2k), due to the stacking fault requiring less out-of-plane distortions to be stable. This is evident from the DFT geometry optimized models, which show more pronounced warping for perfect dislocations (Figure 21 and Figure S18). To summarize, the SFE reduces with increased temperature due to the stacking fault structure of the partial dislocation pair being preferable under high biaxial strain conditions than the perfect dislocation, conditions which arise from the thermal expansion mismatch between graphene and the supporting substrate. Consequently the reduction in SFE with increasing temperature makes the formation of partials more favorable at high temperatures. 

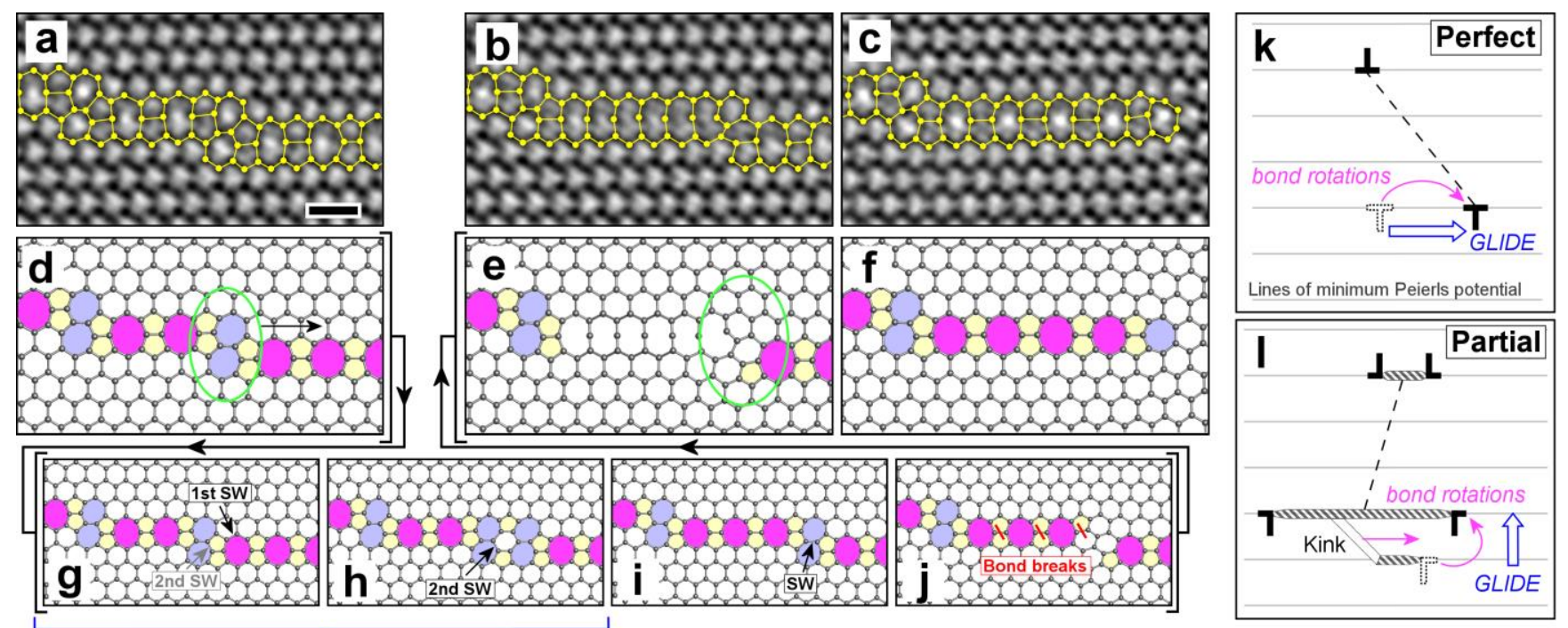

bond rotations for one kink assisted lattice migration

Figure 3. (a-c) AC-TEM sequence of kink assisted glide of a partial dislocation $\left(700{ }^{\circ} \mathrm{C}\right)$. Scale bar $0.5 \mathrm{~nm}$. Time stamps are (a) $t=0 \mathrm{~s}$, (b) $16 \mathrm{~s}$ and (c) $57 \mathrm{~s}$. (d-f) Atomic models of (a-c). (g-j) Atomic models indicating intermediate structures and bond rotations to transition from (d) to (e). Illustrations of dislocation glide in $(\mathrm{k})$ a perfect dislocation, and (l) a partial dislocation.

The dislocation partials were observed to migrate by both glide and climb transformations. Partial dislocation glide was mediated through the zipper-like movement of kinks along the 855 stacking fault between partials, shifting the partial to an adjacent lattice line (Figures 3a-f, Figures S19-S24). ${ }^{1,27}$ We propose that kink migration occurs via a repeating sequence of two Stone-Wales (SW) bond rotations, with each pair of rotations leading to the shift of a single 855 unit to an adjacent lattice line (Figures $3 \mathrm{~g}-$ i). This is supported by tight-binding molecular dynamics (TBMD) (Movie S2) and by DFT, which finds modest energy barriers for the first and second SW rotation of $5.5 \mathrm{eV}$ and $4.6 \mathrm{eV}$, respectively (Figure S20). The kink assisted migration process conserves atoms, and thus a dislocation glide, however the glide direction is orthogonal to that for perfect dislocation glide (Figures $3 \mathrm{k}$ and 1). 

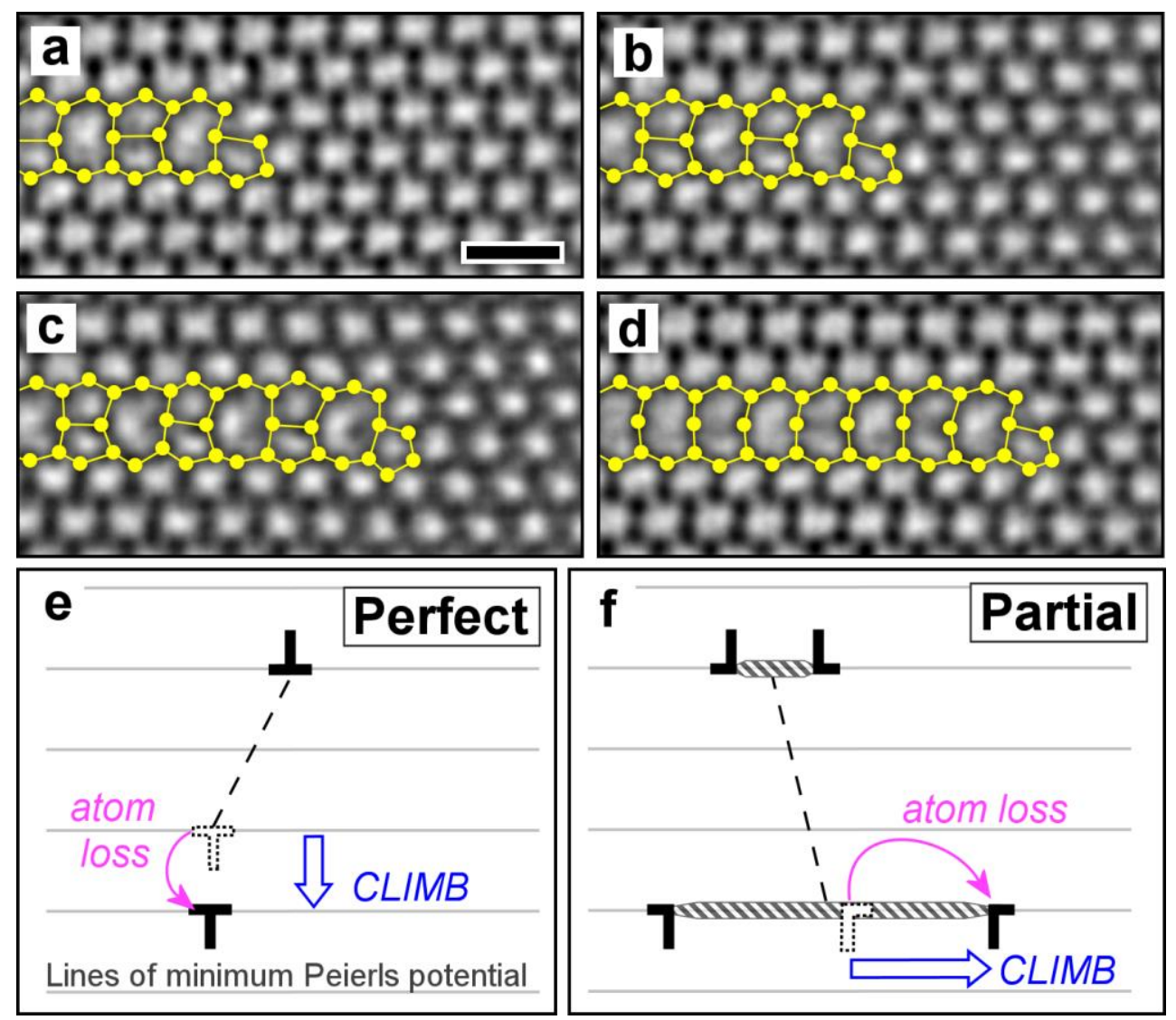

Figure 4. (a-d) AC-TEM sequence of partial dislocation climb through sequential atom removal $\left(700{ }^{\circ} \mathrm{C}\right)$. Time stamps are (a) $t=0 \mathrm{~s}$, (b) $96 \mathrm{~s}$, (c) $113 \mathrm{~s}$ and (d) $141 \mathrm{~s}$. Scale bar is $0.5 \mathrm{~nm}$. Diagrams illustrating dislocation climb through atom loss in (e) a perfect dislocation, and (f) a partial dislocation. Climb directions are reversed for atom addition.

Partial dislocation climb occurs by the removal (or addition) of atoms (Figures 4a-d). Sputtering of carbon from the five-membered rings requires 9 to $12 \mathrm{eV},{ }^{28}$ significantly less than the maximum energy $(\sim 16 \mathrm{eV})$ that can be transferred by an $80 \mathrm{kV}$ electron beam. The removal of atoms separates the partial dislocation cores (Figures S25 and S26) ${ }^{20}$ Climb for perfect dislocations similarly results in an increased separation of the dislocation from its partner (Figure 4e). Quenching of a perfect dislocation can be achieved through successive atom addition climbs until it meets and annihilates with its partner. This occurs when materials are annealed; elevated temperatures increase the climb rate through self-interstitials migrating and coalescing with dislocations, thereby accelerating dislocation removal. ${ }^{1}$ However, when 
annealed a partial pair will instead move towards each other to form a perfect dislocation, rather than moving towards the partner dislocation (Figure 4f). In this way dislocation annihilation is inhibited. Our observations suggest that annealing graphene will result in the dissociation of dislocations into partials, which may then primarily use the lower energy kink migration process (Figure 31) to annihilate.

The formation of partial dislocations in graphene at temperatures of $500{ }^{\circ} \mathrm{C}$ or more will have implications for the development of suitable annealing processes, as our observations show that distinct dislocation diffusion mechanisms become dominant in the high temperature regime. Away from graphene, interestingly our dislocation studies show similarities with the theoretically modeled structures of $30^{\circ}$ partials in silicon, ${ }^{29}$ and we have also found limited evidence for the formation of the $90^{\circ}$ reconstructed partial analogue in graphene (Figure S27) ${ }^{30}$ Similar partial dislocation type mechanisms are expected to occur in other two-dimensional materials as well, ${ }^{31}$ and so it will be of particular interest to investigate if the migration mechanisms identified here are applicable to these other 2D and 3D materials.

\section{Acknowledgements}

JHW thanks the support from the Royal Society and Balliol College, Oxford. AWR has been supported by EPSRC (Platform Grants EP/F048009/1 and EP/K032518/1). Financial support from EPSRC (grants EP/H001972/1 and EP/F028784/1) is acknowledged. G.-D.L. and E.Y. acknowledge support from the Supercomputing Center/Korea Institute of Science and Technology Information with supercomputing resources (KSC-2014-C3-009), from the BK21 plus program, and from the National Research Foundation of Korea (NRF) grant funded by the Korea government (RIAM No. 2010-0012670).

\section{Supporting Information}

CVD graphene growth and transfer details, TEM imaging and processing details, computation modeling details, extra TEM images of dislocations. Supporting Information is available free of charge via the Internet at http://pubs.acs.org.

\section{References}


1. Hull, D.; Bacon, D. J. Introduction to Dislocations; Fourth Edi.; Butterworth-Heinemann: Oxford, 2001.

2. Humphreys, J.; Hatherly, M. Recrystallization and Related Annealing Phenomena; 2nd ed.; Elsevier: Oxford, 2004.

3. Chisholm, M. F.; Kumar, S.; Hazzledine, P. Dislocations in Complex Materials. Science 2005, 307, 701-703.

4. Shibata, N.; Chisholm, M. F.; Nakamura, A.; Pennycook, S. J.; Yamamoto, T.; Ikuhara, Y. Nonstoichiometric Dislocation Cores in Alpha-Alumina. Science 2007, 316, 82-85.

5. Kolar, H.; Spence, J.; Alexander, H. Observation of Moving Dislocation Kinks and Unpinning. Phys. Rev. Lett. 1996, 77, 4031-4034.

6. Wang, Y.; Cheng, X. Deformation Twinning in Nanocrystalline Aluminum. Science 2003, 300, $1275-1277$.

7. Meyer, J. C.; Kisielowski, C.; Erni, R.; Rossell, M. D.; Crommie, M. F.; Zettl, A. Direct Imaging of Lattice Atoms and Topological Defects in Graphene Membranes. Nano Lett. 2008, 8, 3582-6.

8. Carpio, A.; Bonilla, L. Edge Dislocations in Crystal Structures Considered as Traveling Waves in Discrete Models. Phys. Rev. Lett. 2003, 90, 135502.

9. Yazyev, O. V.; Louie, S. G. Topological Defects in Graphene: Dislocations and Grain Boundaries. Phys. Rev. B 2010, 81, 195420.

10. Ding, F.; Jiao, K.; Wu, M.; Yakobson, B. I. Pseudoclimb and Dislocation Dynamics in Superplastic Nanotubes. Phys. Rev. Lett. 2007, 98, 1-4.

11. Lee, G.-D.; Yoon, E.; Hwang, N.-M.; Wang, C.-Z.; Ho, K.-M. Formation and Development of Dislocation in Graphene. Appl. Phys. Lett. 2013, 102, 021603.

12. Warner, J. H.; Margine, E. R.; Mukai, M.; Robertson, A. W.; Giustino, F.; Kirkland, A. I. Dislocation-Driven Deformations in Graphene. Science 2012, 337, 209-12.

13. Lee, G.-D.; Yoon, E.; He, K.; Robertson, A. W.; Warner, J. H. Detailed Formation Processes of Stable Dislocations in Graphene. Nanoscale 2014, 6, 14836-14844.

14. Lehtinen, O.; Kurasch, S.; Krasheninnikov, A. V; Kaiser, U. Atomic Scale Study of the Life Cycle of a Dislocation in Graphene from Birth to Annihilation. Nat. Commun. 2013, 4, 2098.

15. Warner, J. H.; Fan, Y.; Robertson, A. W.; He, K.; Yoon, E.; Lee, G. Do Rippling Graphene at the Nanoscale through Dislocation Addition. Nano Lett. 2013, 13, 4937-44.

16. Butz, B.; Dolle, C.; Niekiel, F.; Weber, K.; Waldmann, D.; Weber, H. B.; Meyer, B.; Spiecker, E. Dislocations in Bilayer Graphene. Nature 2013, 505, 533-537.

17. Ray, I.; Cockayne, D. The Dissociation of Dislocations in Silicon. Proc. R. Soc. London A 1971, $325,543-554$. 
18. Hirashita, N.; Sugiyama, N.; Toyoda, E.; Takagi, S. I. Relaxation Processes in Strained Si Layers on Silicon-Germanium- on-Insulator Substrates. Appl. Phys. Lett. 2005, 86, 1-3.

19. Lahiri, J.; Lin, Y.; Bozkurt, P.; Oleynik, I. I.; Batzill, M. An Extended Defect in Graphene as a Metallic Wire. Nat. Nanotechnol. 2010, 5, 326-9.

20. Chen, J.-H.; Autès, G.; Alem, N.; Gargiulo, F.; Gautam, A.; Linck, M.; Kisielowski, C.; Yazyev, O. V.; Louie, S. G.; Zettl, A. Controlled Growth of a Line Defect in Graphene and Implications for Gate-Tunable Valley Filtering. Phys. Rev. B 2014, 89, 121407.

21. Robertson, A. W.; Lee, G.-D.; He, K.; Yoon, E.; Kirkland, A. I.; Warner, J. H. The Role of the Bridging Atom in Stabilizing Odd Numbered Graphene Vacancies. Nano Lett. 2014, 14, 397280 .

22. Hÿtch, M. J.; Putaux, J.-L.; Pénisson, J.-M. Measurement of the Displacement Field of Dislocations to 0.03 A by Electron Microscopy. Nature 2003, 423, 270-3.

23. Shang, S. L.; Wang, W. Y.; Wang, Y.; Du, Y.; Zhang, J. X.; Patel, a D.; Liu, Z. K. TemperatureDependent Ideal Strength and Stacking Fault Energy of Fcc Ni: A First-Principles Study of Shear Deformation. J. Phys. Condens. Matter 2012, 24, 155402.

24. Sinha, A. K.; Levinstein, H. J.; Smith, T. E. Thermal Stresses and Cracking Resistance of Dielectric Films (SiN, Si3N4, and SiO2) on Si Substrates. J. Appl. Phys. 1978, 49, 2423-2426.

25. Zakharchenko, K. V.; Katsnelson, M. I.; Fasolino, A. Finite Temperature Lattice Properties of Graphene beyond the Quasiharmonic Approximation. Phys. Rev. Lett. 2009, 102, 2-5.

26. Bao, W.; Miao, F.; Chen, Z.; Zhang, H.; Jang, W.; Dames, C.; Lau, C. N. Controlled Ripple Texturing of Suspended Graphene and Ultrathin Graphite Membranes. Nat. Nanotechnol. 2009, $4,562-566$.

27. Cretu, O.; Lin, Y. C.; Suenaga, K. Evidence for Active Atomic Defects in Monolayer Hexagonal Boron Nitride: A New Mechanism of Plasticity in Two-Dimensional Materials. Nano Lett. 2014, 14, 1064-1068.

28. Ding, F.; Jiao, K.; Lin, Y.; Yakobson, B. I. How Evaporating Carbon Nanotubes Retain Their Perfection? Nano Lett. 2007, 7, 681-4.

29. Oyama, N.; Ohno, T. Migration Processes of the $30^{\circ}$ Partial Dislocation in Silicon. Phys. Rev. Lett. 2004, 93, 195502.

30. Bennetto, J.; Nunes, R.; Vanderbilt, D. Period-Doubled Structure for the $90^{\circ}$ Partial Dislocation in Silicon. Phys. Rev. Lett. 1997, 79, 245-248.

31. Liu, Y.; Zou, X.; Yakobson, B. I. Dislocations and Grain Boundaries in Two-Dimensional Boron Nitride. ACS Nano 2012, 6, 7053-7058.

\section{ToC Graphic}




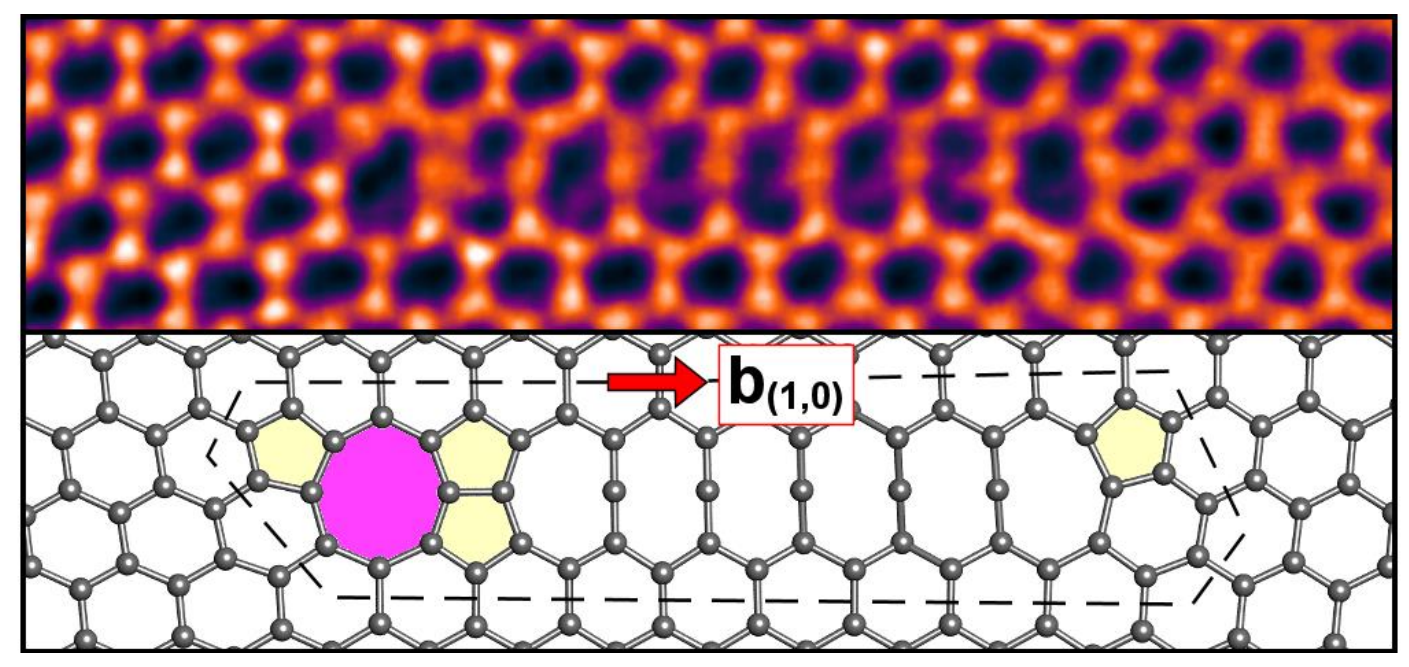

